PROCEEDINGS OF THE

AMERICAN MATHEMATICAL SOCIETY

Volume 134, Number 9, September 2006, Pages 2745-2754

S 0002-9939(06)08402-4

Article electronically published on April 7, 2006

\title{
A GÂTEAUX DIFFERENTIABILITY SPACE THAT IS NOT WEAK ASPLUND
}

\author{
WARREN B. MOORS AND SIVAJAH SOMASUNDARAM
}

(Communicated by Jonathan M. Borwein)

\begin{abstract}
In this paper we construct a Gâteaux differentiability space that is not a weak Asplund space. Thus we answer a question raised by David Larman and Robert Phelps from 1979.
\end{abstract}

\section{INTRODUCTION}

A Banach space $X$ is called a weak Asplund (almost weak Asplund) [Gâteaux differentiability] space if every continuous convex function defined on it is Gâteaux differentiable at the points of a residual (everywhere second category) [dense] subset. While it is easy to see that every weak Asplund space is an almost weak Asplund space and every almost weak Asplund space is a Gâteaux differentiability space it is not so clear whether the reverse implications hold.

In this paper we show that there are in fact some almost weak Asplund spaces that are not weak Asplund. Our considerations are mainly based around the following classes of topological spaces which are defined in terms of minimal uscos. A set-valued mapping $\varphi: X \rightarrow 2^{Y}$ acting between topological spaces $X$ and $Y$ is called an usco mapping if for each $x \in X, \varphi(x)$ is a non-empty compact subset of $Y$ and for each open set $W$ in $Y,\{x \in X: \varphi(x) \subseteq W\}$ is open in $X$. An usco mapping $\varphi: X \rightarrow 2^{Y}$ is called a minimal usco if its graph does not contain, as a proper subset, the graph of any other usco defined on $X$. A topological space $X$ is said to be a Stegall [1] (weakly Stegall [4) space if for every Baire (complete metric) space $B$ and minimal usco $\varphi: B \rightarrow 2^{X}, \varphi$ is single-valued at some point of $B$ or, equivalently, if for every Baire (complete metric) space $B$ and minimal usco $\varphi: B \rightarrow 2^{X}, \varphi$ is single-valued at the points of a residual 2 (everywhere second category [4) subset of $B$. For us, the significance of these spaces stems from the fact that for a Banach space $X$, if $\left(X^{*}\right.$, weak $\left.{ }^{*}\right)$ is a Stegall space, then $X$ is weak Asplund [11, and if $\left(X^{*}\right.$, weak $\left.^{*}\right)$ is a weakly Stegall space, then $X$ is almost weak Asplund [9, Theorem 13] (and so a Gâteaux differentiability space). For more information on weak Asplund spaces, see [2, 3] and [10].

Received by the editors August 31, 2002.

2000 Mathematics Subject Classification. Primary 54C60, 46B20, 54C10.

Key words and phrases. Weak Asplund space, Gâteaux differentiability space, Stegall space.

(C)2006 American Mathematical Society Reverts to public domain 28 years from publication 2745 


\section{The Cantor game And nearly Stegall spaces}

We will say that a $\sigma$-ideal $\mathcal{A}$ of subsets on a topological space $(X, \tau)$ is topologically stable if $h(A) \in \mathcal{A}$ for each homeomorphism $h:(X, \tau) \rightarrow(X, \tau)$ and $A \in \mathcal{A}$. In this paper, $\mathcal{A}$ will always denote a topologically stable $\sigma$-ideal on $\left(\{0,1\}^{\mathbb{N}}, \tau_{p}\right)$, where $\tau_{p}$ denotes the topology of pointwise convergence on $\mathbb{N}$. With this understanding, we can introduce the following notation.

Given a topological space $(X, \tau)$ that is homeomorphic to $\left(\{0,1\}^{\mathbb{N}}, \tau_{p}\right)$ and a topologically stable $\sigma$-ideal $\mathcal{A}$ on $\left(\{0,1\}^{\mathbb{N}}, \tau_{p}\right)$, we shall denote by $\mathcal{A}_{(X, \tau)}$ the induced $\sigma$-ideal on $X$ defined by $\mathcal{A}_{(X, \tau)}:=\left\{h^{-1}(A): A \in \mathcal{A}\right\}$ for some homeomorphism $h:(X, \tau) \rightarrow\left(\{0,1\}^{\mathbb{N}}, \tau_{p}\right)$. (Note: Since $\mathcal{A}$ is topologically stable, the definition of $\mathcal{A}_{(X, \tau)}$ is independent of the particular choice of homeomorphism $h:(X, \tau) \rightarrow\left(\{0,1\}^{\mathbb{N}}, \tau_{p}\right)$.) When there is no ambiguity, we shall simply denote $\mathcal{A}_{(X, \tau)}$ by $\mathcal{A}_{X}$. In terms of this notation we can introduce a stronger notion of topological stability. A $\sigma$-ideal $\mathcal{A}$ on $\left(\{0,1\}^{\mathbb{N}}, \tau_{p}\right)$ is said to be strongly topologically stable if (i) $\mathcal{A}$ is topologically stable and (ii) for each clopen subset $Y$ of $\{0,1\}^{\mathbb{N}}$ that is homeomorphic to $\left(\{0,1\}^{\mathbb{N}}, \tau_{p}\right)$, we have that $\mathcal{A}_{Y} \subseteq \mathcal{A}$.

2.1. The Cantor game. Let $(M, d)$ be a complete metric space without isolated points, let $R$ be a subset of $M$ and let $\mathcal{A}$ be a strongly topologically stable proper $\sigma$-ideal on $\left(\{0,1\}^{\mathbb{N}}, \tau_{p}\right)$. On $M$ we consider the $\mathcal{C}^{\mathcal{A}}(R)$-game played between two players $\alpha$ and $\beta$. Player $\beta$ goes first (always!) and chooses a family $B_{0}:=\left\{B_{0}^{t}\right.$ : $t=\emptyset\}$ consisting of a non-empty open set $B_{0}^{\emptyset}$ with $d$-diam $\left(B_{0}^{\emptyset}\right)<1 / 2^{0}$. Player $\alpha$ must respond to this by choosing a family $A_{0}:=\left\{A_{0}^{t}: t=\emptyset\right\}$ consisting of a non-empty open set $A_{0}^{\emptyset}$ of $B_{0}^{\emptyset}$. Following this player $\beta$ must select another family $B_{1}:=\left\{B_{1}^{t}: t \in\{0,1\}^{1}\right\}$ of non-empty open subsets such that:

(i) $\emptyset=\overline{B_{1}^{0}} \cap \overline{B_{1}^{1}} \subseteq \overline{B_{1}^{0}} \cup \overline{B_{1}^{1}} \subseteq A_{0}^{\emptyset}$ and

(ii) $d$-diam $\left(B_{1}^{t}\right)<1 / 2^{1}$ for all $t \in\{0,1\}^{1}$.

In turn, player $\alpha$ must again respond by selecting a family $A_{1}:=\left\{A_{1}^{t}: t \in\{0,1\}^{1}\right\}$ of non-empty open subsets such that $A_{1}^{t} \subseteq B_{1}^{t}$ for all $t \in\{0,1\}^{1}$.

Continuing this procedure indefinitely the players $\alpha$ and $\beta$ produce a sequence $\left\{\left(A_{n}, B_{n}\right): n \in \omega\right\}$ of ordered pairs of indexed families of non-empty open subsets with $A_{n}:=\left\{A_{n}^{t}: t \in\{0,1\}^{n}\right\}$ and $B_{n}:=\left\{B_{n}^{t}: t \in\{0,1\}^{n}\right\}$ that satisfy the following conditions:

(i) $\emptyset=\overline{B_{n+1}^{t 0}} \cap \overline{B_{n+1}^{t 1}} \subseteq \overline{B_{n+1}^{t 0}} \cup \overline{B_{n+1}^{t 1}} \subseteq A_{n}^{t} \subseteq B_{n}^{t}$ for all $t \in\{0,1\}^{n}$ and

(ii) $d$-diam $\left(B_{n}^{t}\right)<1 / 2^{n}$ for all $t \in\{0,1\}^{n}$.

Such a sequence will be called a play of the $\mathcal{C}^{\mathcal{A}}(R)$-game. We shall declare that $\alpha$ wins a play $\left\{\left(A_{n}, B_{n}\right): n \in \omega\right\}$ of the $\mathcal{C}^{\mathcal{A}}(R)$-game if the set $K \backslash R \in \mathcal{A}_{K}$, where $K:=\bigcap_{n=0}^{\infty} K_{n}$ and $K_{n}:=\bigcup\left\{B_{n}^{t}: t \in\{0,1\}^{n}\right\}$. Otherwise the player $\beta$ is said to have won this play. By a strategy $\sigma$ for the player $\alpha$, we mean a 'rule' that specifies each move of the player $\alpha$ in every possible situation. More precisely a strategy $\sigma:=\left(\sigma_{n}: n \in \omega\right)$ for $\alpha$ is a sequence of functions such that:

(i) $\sigma_{n}\left(B_{0}, B_{1}, \ldots, B_{n}\right):=\left\{\sigma_{n}^{t}\left(B_{0}, B_{1}, \ldots, B_{n}\right): t \in\{0,1\}^{n}\right\}$;

(ii) $\emptyset \neq \sigma_{n}^{t}\left(B_{0}, B_{1}, \ldots, B_{n}\right) \subseteq B_{n}^{t}$ for all $t \in\{0,1\}^{n}$; and

(iii) $\sigma_{n}^{t}\left(B_{0}, B_{1}, \ldots, B_{n}\right)$ is open for all $t \in\{0,1\}^{n}$.

The domain of each function $\sigma_{n}$ is precisely the set of all finite sequences $\left(B_{0}, B_{1}, \ldots, B_{n}\right)$ of indexed families $B_{j}:=\left\{B_{j}^{t}: t \in\{0,1\}^{j}\right\}$ of non-empty open 
subsets that satisfy the following conditions:

(i) $\emptyset=\overline{B_{j+1}^{t 0}} \cap \overline{B_{j+1}^{t 1}} \subseteq \overline{B_{j+1}^{t 0}} \cup \overline{B_{j+1}^{t 1}} \subseteq \sigma_{j}^{t}\left(B_{0}, B_{1}, \ldots, B_{j}\right)$ for all $t \in\{0,1\}^{j}$ and $0 \leq j<n$ and

(ii) $d$-diam $\left(B_{j}^{t}\right)<1 / 2^{j}$ for all $t \in\{0,1\}^{j}$ and $0 \leq j \leq n$.

Such a finite sequence $\left(B_{0}, B_{1}, \ldots, B_{n}\right)$ [infinite sequence $\left.\left(B_{n}: n \in \omega\right)\right]$ is called a partial $\sigma$-play $[\sigma$-play $]$. A strategy $\sigma:=\left(\sigma_{n}: n \in \omega\right)$ for the player $\alpha$ is called a winning strategy if each $\sigma$-play is won by $\alpha$.

To expedite the proof of the following theorem we shall introduce the following notation. Given an indexed family of sets $\left\{A^{t}: t \in\{0,1\}^{n}\right\}$ with $n \in \mathbb{N}$ and $i \in\{0,1\}$ we define

$\left[\left\{A^{t}: t \in\{0,1\}^{n}\right\}\right]^{i}:=\left\{A_{*}^{t^{\prime}}: t^{\prime} \in\{0,1\}^{n-1}\right\}$, where $A_{*}^{t^{\prime}}:=A^{i t^{\prime}}$ for all $t^{\prime} \in\{0,1\}^{n-1}$.

Theorem 1 (Splicing Theorem). Let $(M, d)$ be a complete metric space without isolated points, let $\left\{R_{n}: n \in \mathbb{N}\right\}$ be a family of subsets of $M$ and let $\mathcal{A}$ be a strongly topologically stable proper $\sigma$-ideal on $\left(\{0,1\}^{\mathbb{N}}, \tau_{p}\right)$. If for each $n \in \mathbb{N}$ the player $\alpha$ has a winning strategy in the $\mathcal{C}^{\mathcal{A}}\left(R_{n}\right)$-game played on $M$, then there exists a winning strategy for the player $\alpha$ in the $\mathcal{C}^{\mathcal{A}}\left(\bigcap_{n=1}^{\infty} R_{n}\right)$-game played on $M$.

Proof. For each $k \in \mathbb{N}$, let ${ }^{k} \sigma:=\left({ }^{k} \sigma_{n}: n \in \omega\right)$ be a winning strategy for the player $\alpha$ in the $C^{\mathcal{A}}\left(R_{k}\right)$-game. To prove the theorem we first need to construct a candidate strategy $\sigma:=\left(\sigma_{n}: n \in \omega\right)$ for the player $\alpha$ in the $C^{\mathcal{A}}\left(\cap_{n=1}^{\infty} R_{n}\right)$-game and then show that it is in fact a winning strategy. The idea behind the strategy is simple. If the player $\beta$ selects $B_{0}$, then the player $\alpha$ responds by using ${ }^{1} \sigma_{0}$. Then after player $\beta$ chooses $B_{1}$, player $\alpha$ responds by first applying ${ }^{1} \sigma_{1}$ (once) and then ${ }^{2} \sigma_{0}$ (twice). In general if $\beta$ chooses $B_{n}$ at the $n^{\text {th }}$ stage, then $\alpha$ replies by using ${ }^{1} \sigma_{n}$ ( $2^{0}$ times), ${ }^{2} \sigma_{n-1}$ ( $2^{1}$ times), ${ }^{3} \sigma_{n-2}$ ( $2^{2}$ times) and so on down to applying ${ }^{n+1} \sigma_{0}$ $\left(2^{n}\right.$ times). But we need to be more precise.

First, if $\beta$ chooses $B_{0}:=\left\{B_{0}^{t}: t=\emptyset\right\}$, then $\alpha$ sets ${ }^{0} \Omega_{0}^{\emptyset}:=B_{0}$ and defines $\sigma_{0}\left(B_{0}\right):=\left\{A_{0}^{t}: t=\emptyset\right\}$ where $A_{0}^{\emptyset}:={ }^{1} \sigma_{0}^{\emptyset}\left({ }^{0} \Omega_{0}^{\emptyset}\right)$. Now suppose that ${ }^{k} \Omega_{j}^{s}$ and $\sigma_{j}$ have been defined for each partial $\sigma$-play $\left\{B_{0}, B_{1}, \ldots, B_{j}\right\}$ with $s \in\{0,1\}^{k-1}$ and $1 \leq$ $k \leq j+1 \leq n$ so that:

(i) ${ }^{0} \Omega_{j}^{\emptyset}:=B_{j}$;

(ii) $\left\{{ }^{k-1} \Omega_{k-1}^{s}, \ldots,{ }^{k-1} \Omega_{j-1}^{s},{ }^{k-1} \Omega_{j}^{s}\right\}$ is a partial ${ }^{k} \sigma$-play for each $s \in\{0,1\}^{k-1}$ and $1 \leq k \leq j+1$;

(iii) ${ }^{k} \Omega_{j}^{s i}:=\left[{ }^{k} \sigma_{(j+1-k)}\left({ }^{k-1} \Omega_{k-1}^{s}, \ldots,{ }^{k-1} \Omega_{j-1}^{s},{ }^{k-1} \Omega_{j}^{s}\right)\right]^{i}$ for each $i \in\{0,1\}$, $s \in\{0,1\}^{k-1}$ and $1 \leq k \leq j$;

(iv) $\sigma_{j}\left(B_{0}, B_{1}, \ldots, B_{j}\right):=\left\{A_{j}^{t}: t \in\{0,1\}^{j}\right\}$, where $A_{j}^{t}:={ }^{j+1} \sigma_{0}^{\emptyset}\left({ }^{j} \Omega_{j}^{t}\right)$ for each $t \in\{0,1\}^{j}$.

If the $n^{\text {th }}$ move of $\beta$ is $B_{n}:=\left\{B_{n}^{t}: t \in\{0,1\}^{n}\right\}$, then $\alpha$ responds in the following way:

(i) First $\alpha$ sets ${ }^{0} \Omega_{n}^{\emptyset}:=B_{n}$ and then inductively defines.

(ii) ${ }^{k} \Omega_{n}^{s i}:=\left[{ }^{k} \sigma_{(n+1-k)}\left({ }^{k-1} \Omega_{k-1}^{s}, \ldots,{ }^{k-1} \Omega_{n-1}^{s},{ }^{k-1} \Omega_{n}^{s}\right)\right]^{i}$ for each $i \in\{0,1\}$, $s \in\{0,1\}^{k-1}$ and $1 \leq k \leq n$.

(iii) Then $\alpha$ observes that with this definition, for each $s \in\{0,1\}^{k-1}$ and $1 \leq k \leq n+1,\left\{{ }^{k-1} \Omega_{k-1}^{s}, \ldots,{ }^{k-1} \Omega_{n-1}^{s},{ }^{k-1} \Omega_{n}^{s}\right\}$ is a partial ${ }^{k} \sigma$-play.

(iv) Finally $\alpha$ defines $\sigma_{n}\left(B_{0}, B_{1}, \ldots, B_{n}\right):=\left\{A_{n}^{t}: t \in\{0,1\}^{n}\right\}$ where $A_{n}^{t}:=$ ${ }^{n+1} \sigma_{0}^{\emptyset}\left({ }^{n} \Omega_{n}^{t}\right)$ for all $t \in\{0,1\}^{n}$. 
This completes the definition of $\sigma:=\left(\sigma_{n}: n \in \omega\right)$. It remains to show that $\sigma:=$ $\left(\sigma_{n}: n \in \omega\right)$ is indeed a winning strategy for the player $\alpha$ in the $C^{\mathcal{A}}\left(\cap_{n=1}^{\infty} R_{n}\right)$-game. To accomplish this, it will be sufficient to show that for any $\sigma$-play $\left(B_{n}: n \in \omega\right)$ and $m \in \mathbb{N}, K \backslash R_{m} \in \mathcal{A}_{K}$ where $K:=\bigcap_{n=1}^{\infty} K_{n}$ and $K_{n}:=\bigcup\left\{B_{n}^{t}: t \in\{0,1\}^{n}\right\}$ for all $n \in \omega$. To this end, let $\left(B_{n}: n \in \omega\right)$ be a fixed $\sigma$-play and let $m$ be a fixed member of $\mathbb{N}$. For each $s \in\{0,1\}^{m-1}$ let

$$
K^{s}:=\bigcap_{j \geq(m-1)}\left\{\bigcup\left\{B_{j}^{t}: t \in\{0,1\}^{j} \text { and } s \leq t\right\}\right\}=\bigcap_{j \geq(m-1)}{ }^{m-1} \Omega_{j}^{s} .
$$

Now $\left\{{ }^{m-1} \Omega_{j}^{s}: j \geq m-1\right\}$ is a ${ }^{m} \sigma$-play and so

$$
\left(\bigcap_{j \geq(m-1)}{ }^{m-1} \Omega_{j}^{s}\right) \backslash R_{m}=K^{s} \backslash R_{m} \in \mathcal{A}_{K^{s}} \subseteq \mathcal{A}_{K},
$$

since for each $s \in\{0,1\}^{m-1}, K^{s}$ is homeomorphic to $\left(\{0,1\}^{\mathbb{N}}, \tau_{p}\right)$ and a clopen subspace of $K$. Therefore,

$$
\begin{aligned}
K \backslash R_{m} & =\bigcap\left\{K_{j}: j \geq m-1\right\} \backslash R_{m}=\bigcup\left\{K^{s}: s \in\{0,1\}^{m-1}\right\} \backslash R_{m} \\
& =\bigcup\left\{K^{s} \backslash R_{m}: s \in\{0,1\}^{m-1}\right\} \in \mathcal{A}_{K} .
\end{aligned}
$$

This shows that $\sigma:=\left(\sigma_{n}: n \in \omega\right)$ is a winning strategy for the player $\alpha$ in the $C^{\mathcal{A}}\left(\cap_{n=1}^{\infty} R_{n}\right)$-game played on $M$.

Given a strongly topologically stable proper $\sigma$-ideal $\mathcal{A}$ on $\left(\{0,1\}^{\mathbb{N}}, \tau_{p}\right)$ and a topological space $X$ we shall say that $X$ is nearly Stegall with respect to $\mathcal{A}$ if, for every complete metric space $M$ without isolated points and minimal usco $\varphi: M \rightarrow$ $2^{X}$, there exists a winning strategy for the player $\alpha$ in the $\mathcal{C}^{\mathcal{A}}(R)$-game played on $M$, where $R$ is the set of points at which $\varphi$ is single-valued.

It is not difficult to show that for any strongly topologically stable proper $\sigma$ ideal $\mathcal{A}$ on $\left(\{0,1\}^{\mathbb{N}}, \tau_{p}\right)$ we have the following: if $X$ is a Stegall space, then $X$ is nearly Stegall with respect to $\mathcal{A}$; if $X$ is nearly Stegall with respect to $\mathcal{A}$, then $X$ is weakly Stegall.

The following theorem is now a simple consequence of the Splicing Theorem.

Theorem 2. Let $\rho$ be a metric on a topological space $X$ and let $\mathcal{A}$ be a strongly topologically stable proper $\sigma$-ideal on $\left(\{0,1\}^{\mathbb{N}}, \tau_{p}\right)$. Then $X$ is a nearly Stegall space with respect to $\mathcal{A}$ if (and only if) for each $\varepsilon>0$, each complete metric space $M$ without isolated points and each minimal usco $\varphi: M \rightarrow 2^{X}$, there exists a winning strategy for the player $\alpha$ in the $\mathcal{C}^{\mathcal{A}}\left(R_{\varepsilon}\right)$-game, where $R_{\varepsilon}:=\{m \in M$ : $\rho$-diam $[\varphi(m)] \leq \varepsilon\}$.

In the proof of our main result we will need the following basic properties of nearly Stegall spaces. Since the proofs of these assertions are similar to those given in Theorem 3.1.5 of [2], we shall not give them here.

Theorem 3. Let $(X, \tau)$ and $(Y, \tau)$ be topological spaces and let $\mathcal{A}$ denote a strongly topologically stable proper $\sigma$-ideal on $\left(\{0,1\}^{\mathbb{N}}, \tau_{p}\right)$.

(i) Let $f: X \rightarrow Y$ be a perfect mapping onto $Y$. If $X$ is a nearly Stegall space with respect to $\mathcal{A}$, then $Y$ is a nearly Stegall space with respect to $\mathcal{A}$.

(ii) Let $\left\{X_{n}: n \in \mathbb{N}\right\}$ be a cover of $X$. If each $X_{n}$ is a closed subset of $X$ and is a nearly Stegall space with respect to $\mathcal{A}$, then $X$ is a nearly Stegall space with respect to $\mathcal{A}$. 
(iii) If each $\left\{X_{n}: n \in \mathbb{N}\right\}$ is a nearly Stegall space with respect to $\mathcal{A}$, then $\prod_{n=1}^{\infty} X_{n}$ is a nearly Stegall space with respect to $\mathcal{A}$.

\section{A weakly Stegall Space that is not weak Asplund}

Let $A$ be an arbitrary subset of $(0,1)$ and let

$$
K_{A}:=[(0,1] \times\{0\}] \cup[(\{0\} \cup A) \times\{1\}] .
$$

If we equip this set with the order topology generated by the lexicographical (dictionary) ordering (i.e., $\left(s_{1}, s_{2}\right) \leq\left(t_{1}, t_{2}\right)$ if and only if either $s_{1}<t_{1}$ or $s_{1}=t_{1}$ and $\left.s_{2} \leq t_{2}\right)$, then with this topology $K_{A}$ is a compact Hausdorff space [5. Proposition $2]$. In the special case of $A=(0,1), K_{A}$ reduces to the well-known "double arrow" space.

For any subset $A$ of $(0,1)$ we shall denote by $B V_{A}[0,1]$ the space of all realvalued functions of bounded variation on $[0,1]$ that are right continuous at the points of $(0,1) \backslash A$ and map 0 to 0 . We will consider this space endowed with the total variation norm, i.e., for each $\alpha \in B V_{A}[0,1]$,

$$
\|\alpha\|:=\operatorname{Var}(\alpha)=\sup \left\{\sum_{k=1}^{n}\left|\alpha\left(t_{k}\right)-\alpha\left(t_{k-1}\right)\right|:\right.
$$

$$
\left.\left\{t_{k}: 0 \leq k \leq n\right\} \text { is a partition of }[0,1]\right\} .
$$

For a non-empty subset $A$ of $[0,1]$ we shall denote by $\tau_{A}$ the topology (on $\left.B V_{A}[0,1]\right)$ of pointwise convergence on $A \cup\{1\}$. If $A$ is dense in $[0,1]$, then $\tau_{A}$ is a Hausdorff topology. Moreover the closed unit ball in $B V_{A}[0,1]$ (with respect to the total variation norm) is $\tau_{A}$-compact. The following proposition reveals the relationship between the spaces $C\left(K_{A}\right)$ and $B V_{A}[0,1]$.

Proposition 1 ([6, Corollary 1$])$. For a dense subset $A$ of $(0,1)$, the closed unit ball $B_{B V_{A}[0,1]}$ in $B V_{A}[0,1]$ with the $\tau_{A}$-topology is homeomorphic to $\left(B_{C\left(K_{A}\right)^{*}}\right.$, weak $\left.k^{*}\right)$.

A more detailed analysis of Proposition 1 may be found in [9, Theorem 7]. Next, we give some technical results that will be needed in our main theorem.

Lemma 1 (6, Lemma 2]). Let $\varphi: X \rightarrow 2^{Y}$ be a minimal usco acting between topological spaces $X$ and $Y$ and let $f: Y \rightarrow \mathbb{R}$ be a continuous function. Then there is a residual set $R$ in $X$ such that the composition mapping $f \circ \varphi: X \rightarrow 2^{\mathbb{R}}$ defined by $(f \circ \varphi)(x):=\{f(y): y \in \varphi(x)\}$ is single-valued at the points of $R$.

The following result is a consequence of Proposition 5.1 and Proposition 5.3 in 12 .

Proposition 2 ([9, Proposition 1]). Let $\varphi: B \rightarrow 2^{X}$ be a minimal usco acting from a Baire space $B$ into a topological space $X$. If $K$ is a Borel set and $\varphi^{-1}(K):=\{b \in B: \varphi(b) \cap K \neq \emptyset\}$ is second category, then there exist a non-empty open subset $U$ of $B$ and a dense $G_{\delta}$ subset $G$ of $U$ such that $\varphi(G) \subseteq K$.

For each $\alpha \in M_{A}[0,1]$, the set of all non-decreasing functions in $B_{B V_{A}[0,1]}$, and each $m \in \mathbb{N}$ we define

$$
S(\alpha, m):=\left\{t \in[0,1]: \alpha\left(t^{+}\right)-\alpha\left(t^{-}\right) \geq 1 / m\right\}
$$

where $\alpha\left(t^{+}\right):=\lim _{t^{\prime} \rightarrow t^{+}} \alpha\left(t^{\prime}\right)$ for $t \in[0,1), \alpha\left(1^{+}\right):=\alpha(1), \alpha\left(t^{-}\right):=\lim _{t^{\prime} \rightarrow t^{-}} \alpha\left(t^{\prime}\right)$ for $t \in(0,1]$ and $\alpha\left(0^{-}\right):=\alpha(0)=0$. 
Lemma 2 (Basic Lemma). Let $A$ be a dense subset of $(0,1)$, let $\alpha \in M_{A}[0,1]$, let $m \in \mathbb{N}$ and let $0 \leq a<b \leq 1$. If $S(\alpha, m) \cap[a, b]=\emptyset$, then there exists $a$ neighbourhood $U(\alpha)$ of $\alpha$ in $\left(M_{A}[0,1], \tau_{A}\right)$ such that $S(\beta, m) \cap[a, b]=\emptyset$ for each $\beta \in U(\alpha)$.

Corollary 1. Let $A$ be a dense subset of $(0,1)$, let $\alpha \in M_{A}[0,1]$, let $m \in \mathbb{N}$ and let $U$ be an open subset of $[0,1]$. If $S(\alpha, m) \subseteq U$, then there exists a neighbourhood $U(\alpha)$ of $\alpha$ in $\left(M_{A}[0,1], \tau_{A}\right)$ such that $S(\beta, m) \subseteq U$ for each $\beta \in U(\alpha)$.

We are now in a position to present our main theorem.

Theorem 4. Let $\mathcal{A}$ be a strongly topologically stable proper $\sigma$-ideal on $\left(\{0,1\}^{\mathbb{N}}, \tau_{p}\right)$ and let $A$ be a dense subset of $(0,1)$ such that $C \cap A \in \mathcal{A}_{C}$ for each set $C \subseteq[0,1]$ that is homeomorphic to $\left(\{0,1\}^{\mathbb{N}}, \tau_{p}\right)$. Then $\left(B V_{A}[0,1], \tau_{A}\right)$ is nearly Stegall with respect to $\mathcal{A}$. In particular, $\left(B V_{A}[0,1], \tau_{A}\right)$ is weakly Stegall.

Proof. First, let us note that by Theorem 3, part(ii), we need only show that the closed unit ball $B_{B V_{A}[0,1]}$ of $B V_{A}[0,1]$ is nearly Stegall with respect to $\mathcal{A}$. In fact, we need only show that the ( $\tau_{A}$-compact) set $M_{A}[0,1]$ of all non-decreasing functions in $B_{B V_{A}[0,1]}$, endowed with the $\tau_{A}$-topology, is nearly Stegall with respect to $\mathcal{A}$. Since if $M_{A}[0,1]$ is nearly Stegall with respect to $\mathcal{A}$, then by Theorem 3 , part(iii), $M_{A}[0,1] \times M_{A}[0,1]$ is nearly Stegall with respect to $\mathcal{A}$. However, by the Jordan decomposition theorem $B_{B V_{A}[0,1]} \subseteq \triangle\left(M_{A}[0,1] \times M_{A}[0,1]\right)$, where $\triangle: M_{A}[0,1] \times M_{A}[0,1] \rightarrow B V_{A}[0,1]$ is defined by $\triangle(f, g):=f-g$. Hence the result follows from Theorem 3 , part(i), since $\triangle$ is a perfect mapping.

For any $\alpha, \beta$ in $M_{A}[0,1]$ we define

$$
\rho_{I}(\alpha, \beta):=\sum_{n=1}^{\infty}\left|(\alpha-\beta)\left(a_{n}\right)\right| / 2^{n} \text { and } \rho_{J}(\alpha, \beta):=\sum_{t \in A}\left|(\alpha-\beta)\left(t^{+}\right)-(\alpha-\beta)(t)\right|
$$

where $a_{1}:=1$ and $\left\{a_{n}: n \geq 2\right\} \subseteq A$ is dense in $[0,1]$.

Note: $\left\{t \in A:\left|(\alpha-\beta)\left(t^{+}\right)-(\alpha-\beta)(t)\right|>0\right\}$ is at most countable. Then we define $\rho(\alpha, \beta):=\rho_{I}(\alpha, \beta)+\rho_{J}(\alpha, \beta)$. With a little thought it should be clear that $\rho$ defines a metric on the set $M_{A}[0,1]$. We now proceed via Theorem 2. To this end, let $\varepsilon>0$, let $M$ be a complete metric space without isolated points, let $\varphi: M \rightarrow 2^{M_{A}[0,1]}$ be a minimal usco and let $R_{\varepsilon}:=\{x \in M: \rho-\operatorname{diam}[\varphi(x)] \leq \varepsilon\}$.

Step 1. It is not too difficult to check that $\rho_{I}$ is a continuous pseudo-metric on $M_{A}[0,1]$, i.e., for each $\alpha \in M_{A}[0,1]$ and $r>0$ the set $\left\{\beta \in M_{A}[0,1]: \rho_{I}(\alpha, \beta)<r\right\}$ is $\tau_{A}$-open in $M_{A}[0,1]$. Hence it follows that $\rho_{I}$ "fragments" $M_{A}[0,1]$. In particular this means that there is a residual set $R \subseteq M$ such that $\rho_{I}$ - $\operatorname{diam}[\varphi(x)]=0$ at each point $x \in R$ (see the proof of Theorem 5.1.11 in [2]). One immediate consequence of this is that for each $x \in R$, we may unambiguously refer to the left-hand and righthand limits of $\varphi(x)$, since if $\alpha, \beta \in \varphi(x)$, then both the left-hand and right-hand limits of $\alpha$ and $\beta$ coincide on $[0,1]$.

Step 2. In this step we decompose the space $M_{A}[0,1]$ into countably many parts, $\left\{M_{m, n, F}:(m, n, F) \in \mathbb{N}^{2} \times \mathcal{F}\right\}$, but first we introduce some notation. For each $\alpha \in M_{A}[0,1]$ and $m \in \mathbb{N}$ we define

$$
L^{1}(\alpha, m):=\sum_{t \in S(\alpha, m)}\left[\alpha\left(t^{+}\right)-\alpha\left(t^{-}\right)\right] .
$$


The notation $S(\alpha, \infty)$ and $L^{1}(\alpha, \infty)$ will have the expected meaning. For each $m \in \mathbb{N}$, we define $M_{m}:=\left\{\alpha \in M_{A}[0,1]: L^{1}(\alpha, m)>L^{1}(\alpha, \infty)-\varepsilon / 2\right\}$ and for each partition $P:=\left\{t_{k}: 0 \leq k \leq n\right\}$ of $[0,1]$, we let $I_{k}(P):=\left[t_{k-1}, t_{k}\right]$, for all $1 \leq k \leq n$. Then for each $n \in \mathbb{N}$, we let $P_{n}$ denote the uniform $1 / n$-partition of $[0,1]$ and we define

$$
\begin{aligned}
& M_{m, n}:=\left\{\alpha \in M_{m}: P_{n}^{*} \cap S(\alpha, m)=\emptyset\right. \text { and } \\
& \left.\quad \operatorname{card}\left[S(\alpha, m) \cap I_{k}\left(P_{n}\right)\right] \leq 1 \text { for } k \in\{1,2, \ldots, n\}\right\}
\end{aligned}
$$

where $P_{n}^{*}:=P_{n} \backslash\left\{t_{0}, t_{n}\right\}$. One can now check that $\bigcup\left\{M_{m, n}:(m, n) \in \mathbb{N}^{2}\right\}=$ $M_{A}[0,1]$. Now with $m$ and $n$ fixed, we further decompose $M_{A}[0,1]$ as follows. For each fixed non-empty subset $F \subseteq\{1,2, \ldots, n\}$, we consider the set

$$
M_{m, n, F}:=\left\{\alpha \in M_{m, n}: \operatorname{card}\left[I_{k}\left(P_{n}\right) \cap S(\alpha, m)\right]=1 \text { if and only if } k \in F\right\} .
$$

If we let $\mathcal{F}$ denote the family of all subsets of $\{1,2, \ldots, n\}$, then $\mathcal{F}$ is finite. Hence $\left\{M_{m, n, F}:(m, n, F) \in \mathbb{N}^{2} \times \mathcal{F}\right\}$ is a countable decomposition of $M_{A}[0,1]$.

Step 3. We claim that for each $(m, n, F) \in \mathbb{N}^{2} \times \mathcal{F}, M_{m, n, F}$ is a Borel subset of $\left(M_{A}[0,1], \tau_{A}\right)$. We begin by noting that with the aid of Lemma 2 it is not difficult to justify the fact that for each $m \in \mathbb{N}$, the mapping $\alpha \mapsto L^{1}(\alpha, m)$ is upper semi-continuous on $\left(M_{A}[0,1], \tau_{A}\right)$. Now $L^{1}(\alpha, \infty)=\lim _{m \rightarrow \infty} L^{1}(\alpha, m)$, therefore the mapping $\alpha \mapsto L^{1}(\alpha, \infty)$ is Borel measurable on $\left(M_{A}[0,1], \tau_{A}\right)$ and hence for each $m \in \mathbb{N}, M_{m}$ is a Borel subset of $\left(M_{A}[0,1], \tau_{A}\right)$. Next, for each partition $P:=\left\{t_{k}: 0 \leq k \leq n\right\}$ of $[0,1]$ and $F \subseteq\{1,2, \ldots, n\}$, let

$$
M_{m}(P, F):=\left\{\alpha \in M_{m}: S(\alpha, m) \cap P^{*}=\emptyset \text { and } S(\alpha, m) \subseteq \bigcup_{k \in F} I_{k}(P)\right\}
$$

where $P^{*}:=P \backslash\left\{t_{0}, t_{n}\right\}$. By again appealing to Lemma 2 it should be easy to see that for each partition $P$ of $[0,1]$ and subset $F \subseteq\{1,2, \ldots, n\}, M_{m}(P, F)$ is $\tau_{A^{-}}$open in $M_{m}$. Now, for each partition $P$ on $[0,1]$ and subset $F \subseteq\{1,2, \ldots, n\}$ let

$$
M_{m}^{*}(P, F):=\left\{\alpha \in M_{m}(P, F): S(\alpha, m) \cap I_{k}(P) \neq \emptyset \text { if and only if } k \in F\right\} ;
$$

then $M_{m}^{*}(P, F)=M_{m}(P, F) \backslash \bigcup\left\{M_{m}\left(P, F^{\prime}\right): F^{\prime}\right.$ is a proper subset of $\left.F\right\}$. Thus $M_{m}^{*}(P, F)$ is a Borel subset of $M_{m}(P, F)$ and hence a Borel subset of $\left(M_{A}[0,1], \tau_{A}\right)$. With $m, P, F$ and $\delta>0$ fixed define

$$
M_{m}^{*}(P, F, \delta):=\left\{\alpha \in M_{m}^{*}(P, F): \operatorname{diam}\left[S(\alpha, m) \cap I_{k}(P)\right]<\delta \text { for all } k \in F\right\} .
$$

Now from Corollary 1 it is clear that for each $\delta>0, M_{m}^{*}(P, F, \delta)$ is relatively open in $M_{m}^{*}(P, F)$. Therefore,

$$
\begin{aligned}
M_{m, n, F} & =\left\{\alpha \in M_{m, n}: \operatorname{card}\left[I_{k}\left(P_{n}\right) \cap S(\alpha, m)\right]=1 \text { if and only if } k \in F\right\} \\
& =\bigcap\left\{M_{m}^{*}\left(P_{n}, F, 1 / j\right): j \in \mathbb{N}\right\}
\end{aligned}
$$

which is a $G_{\delta^{-}}$-subset of $M_{m}^{*}\left(P_{n}, F\right)$ and so a Borel subset of $\left(M_{A}[0,1], \tau_{A}\right)$.

Step 4. In this step we describe the strategy for the player $\alpha$ in the $C^{\mathcal{A}}\left(R_{\epsilon}\right)$ game played on $M$. First, suppose $\beta$ chooses $B_{0}:=\left\{B_{0}^{t}: t=\emptyset\right\}$. Then for each $(m, n, F) \in \mathbb{N}^{2} \times \mathcal{F}$ we define

$$
R_{m, n, F}:=\left\{x \in B_{0}^{\emptyset}: \varphi(x) \cap M_{m, n, F} \neq \emptyset\right\} .
$$


Now since $B_{0}^{\emptyset}=\bigcup\left\{R_{m, n, F}:(m, n, F) \in \mathbb{N}^{2} \times \mathcal{F}\right\}$, it follows that for some $\left(m^{\prime}, n^{\prime}, F^{\prime}\right) \in \mathbb{N}^{2} \times \mathcal{F}, R_{m^{\prime}, n^{\prime}, F^{\prime}}$ is of second category in $B_{0}^{\emptyset}$ and so by Proposition 2 there exists a non-empty open subset $U$ of $B_{0}$ and a dense $G_{\delta}$ subset $G$ of $U \cap R$ such that $\varphi(G) \subseteq M_{m^{\prime}, n^{\prime}, F^{\prime}}$. For each $k \in F^{\prime} \subseteq\left\{1,2 \ldots, n^{\prime}\right\}$ we define the function $g_{k}: G \rightarrow[0,1]$ by $g_{k}(x):=S\left(\varphi(x), m^{\prime}\right) \cap I_{k}\left(P_{n^{\prime}}\right)$. Note: this definition is sensible since for each $x \in G$ and $\alpha, \beta \in \varphi(x), S\left(\alpha, m^{\prime}\right)=S\left(\beta, m^{\prime}\right)$. It now follows from the $\tau_{A^{-}}$-upper semicontinuity of $\varphi$, Corollary 1 and the definition of $M_{m^{\prime}, n^{\prime}, F^{\prime}}$ that each $g_{k}$ is continuous on $G$. Player $\alpha$ now selects a non-empty open subset $A_{0}^{\emptyset}$ of $U \subseteq B_{0}^{\emptyset}$ such that for each $k \in F^{\prime}$ either $g_{k}$ is constant on $A_{0}^{\emptyset} \cap G$ or $g_{k}$ is not constant on $W \cap G$ for any non-empty open subset $W$ of $A_{0}^{\emptyset}$. Then $\alpha$ sets

$$
\begin{gathered}
F_{1}^{\prime}:=\left\{k \in F^{\prime}: g_{k} \text { is constant on } A_{0}^{\emptyset} \cap G \text { and } g_{k}\left(A_{0}^{\emptyset}\right) \cap A=\emptyset\right\} ; \\
F_{2}^{\prime}:=\left\{k \in F^{\prime}: g_{k} \text { is constant on } A_{0}^{\emptyset} \cap G \text { and } g_{k}\left(A_{0}^{\emptyset}\right) \subseteq A\right\} ; \quad \text { and }
\end{gathered}
$$

$F_{3}^{\prime}:=\left\{k \in F^{\prime}: g_{k}\right.$ is not constant on $G \cap W$ for any non-void open set $\left.W \subseteq A_{0}^{\emptyset}\right\}$.

For each $k \in F_{2}^{\prime}$, $\alpha$ defines $t_{k}:=g_{k}(x)$ for $x \in A_{0}^{\emptyset} \cap G$. Then by Lemma 1 there exists a residual set $R^{*} \subseteq G \cap A_{0}^{\emptyset}$ such that each of the uscos $\left(t_{k} \circ \varphi\right): G \cap A_{0}^{\emptyset} \rightarrow 2^{\mathbb{R}}$ defined by $\left(t_{k} \circ \varphi\right)(x):=\left\{\alpha\left(t_{k}\right): \alpha \in \varphi(x)\right\}$ are single-valued on $R^{*}$. Next $\alpha$ chooses a sequence of dense open sets $\left\{O_{n}: n \in \mathbb{N}\right\}$ of $A_{0}^{\emptyset}$ such that $\bigcap_{n=1}^{\infty} O_{n} \subseteq R^{*} \subseteq G \cap A_{0}^{\emptyset}$ and notes that for each $k \in F_{1}^{\prime} \cup F_{2}^{\prime}$ and $x \in R^{*}, \varphi(x)\left(g_{k}(x)\right)$ is a singleton.

Then $\alpha$ defines $\sigma_{0}\left(B_{0}\right):=\left\{A_{0}^{t}: t=\emptyset\right\}$. In general if $\beta$ selects $B_{n}:=\left\{B_{n}^{t}:\right.$ $\left.t \in\{0,1\}^{n}\right\}$, then $\alpha$ responds by choosing non-empty open subsets $A_{n}^{t} \subseteq B_{n}^{t} \cap O_{n}$ such that $g_{k}\left(A_{n}^{t}\right) \cap g_{k}\left(A_{n}^{t^{\prime}}\right)=\emptyset$ for all $k \in F_{3}^{\prime}$ and all $t, t^{\prime} \in\{0,1\}^{n}$ with $t \neq t^{\prime}$. Then $\alpha$ defines $\sigma_{n}\left(B_{0}, B_{1}, \ldots, B_{n}\right):=\left\{A_{n}^{t}: t \in\{0,1\}^{n}\right\}$. This completes the definition of $\sigma:=\left(\sigma_{n}: n \in \omega\right)$.

Step 5. We now show that the just described strategy $\sigma$ is a winning strategy for the player $\alpha$ in the $C^{\mathcal{A}}\left(R_{\varepsilon}\right)$-game. To this end, let $K:=\bigcap_{n=0}^{\infty}\left\{\bigcup\left\{B_{n}^{t}\right.\right.$ : $\left.\left.t \in\{0,1\}^{n}\right\}\right\} \subseteq R^{*}$ and, for each $k \in F_{3}^{\prime}$, let

$$
\mathcal{A}(k):=g_{k}^{-1}(A) \cap K=g_{k}^{-1}\left(g_{k}(K) \cap A\right) .
$$

Since $g_{k}(K)$ is homeomorphic to $\left(\{0,1\}^{\mathbb{N}}, \tau_{p}\right), g_{k}(K) \cap A \in \mathcal{A}_{g_{k}(K)}$ and so $\mathcal{A}(k) \in \mathcal{A}_{K}$. We claim that $K \backslash R_{\varepsilon} \subseteq \bigcup\left\{\mathcal{A}(k): k \in F_{3}^{\prime}\right\} \in \mathcal{A}_{K}$. To see this, first note that it is sufficient to show that $\rho_{J}$-diam $[\varphi(x)] \leq \varepsilon$ for each $x \in K \backslash \bigcup\left\{\mathcal{A}(k): k \in F_{3}^{\prime}\right\}$. Now fix $x_{0} \in K \backslash \bigcup\left\{\mathcal{A}(k): k \in F_{3}^{\prime}\right\}$ and consider $\alpha, \beta \in \varphi\left(x_{0}\right)$; then

$$
\rho_{J}(\alpha, \beta)=\sum_{t \in A}\left|(\alpha-\beta)\left(t^{+}\right)-(\alpha-\beta)(t)\right|=\sum_{t \in S(\alpha, \infty)}\left|(\alpha-\beta)\left(t^{+}\right)-(\alpha-\beta)(t)\right| .
$$

However, if $t \in S\left(\alpha, m^{\prime}\right)$, then $\left|(\alpha-\beta)\left(t^{+}\right)-(\alpha-\beta)(t)\right|=0$ because $\alpha\left(t^{+}\right)=\beta\left(t^{+}\right)$ (by Step 1) and $\alpha(t)=\beta(t)$ since $\alpha\left(g_{k}\left(x_{0}\right)\right)=\beta\left(g_{k}\left(x_{0}\right)\right)$ for all $k \in F^{\prime}$. On the other hand, if we write $S_{\text {tail }}:=S(\alpha, \infty) \backslash S\left(\alpha, m^{\prime}\right)$, then we have

$$
\begin{aligned}
\sum_{t \in S_{\text {tail }}}\left|(\alpha-\beta)\left(t^{+}\right)-(\alpha-\beta)(t)\right| & \leq \sum_{t \in S_{\text {tail }}} \alpha\left(t^{+}\right)-\alpha(t)+\sum_{t \in S_{\text {tail }}} \beta\left(t^{+}\right)-\beta(t) \\
& \leq \sum_{t \in S_{\text {tail }}} \alpha\left(t^{+}\right)-\alpha\left(t^{-}\right)+\sum_{t \in S_{\text {tail }}} \beta\left(t^{+}\right)-\beta\left(t^{-}\right) \\
& <\varepsilon / 2+\varepsilon / 2=\varepsilon .
\end{aligned}
$$

Thus $\rho(\alpha, \beta) \leq \varepsilon$ and so $\rho$ - $\operatorname{diam}\left[\varphi\left(x_{0}\right)\right] \leq \varepsilon$; which completes the proof.

To apply Theorem 4 we need to consider some small subsets of $\mathbb{R}$. 
Lemma 3. There exist a dense second category subset $A$ of $(0,1)$ and a strongly topologically stable proper $\sigma$-ideal $\mathcal{A}$ on $\left(\{0,1\}^{\mathbb{N}}, \tau_{p}\right)$ such that $C \cap A \in \mathcal{A}_{C}$ for each set $C \subseteq[0,1]$ that is homeomorphic to $\left(\{0,1\}^{\mathbb{N}}, \tau_{p}\right)$.

Proof. Let $\kappa$ be the least ordinal of cardinality $2^{\aleph_{0}}$, let $\left\{\left(f_{n}^{\alpha}: n \in \mathbb{N}\right): \alpha<\kappa\right\}$ be an enumeration of all the sequences of continuous one-to-one functions from $\left(\{0,1\}^{\mathbb{N}}, \tau_{p}\right)$ into $[0,1]$ and let $\left\{E^{\alpha}: \alpha<\kappa\right\}$ be an enumeration of all the nonmeager Borel subsets of $(0,1)$. Inductively, we may choose

$$
\begin{aligned}
a_{\alpha} \in E^{\alpha} \backslash\left\{f_{n}^{\beta}\left(x_{\beta}\right): n \in \mathbb{N} \text { and } \beta<\alpha\right\} \quad \text { and } \quad x_{\alpha} \in\{0,1\}^{\mathbb{N}} \text { such that } \\
\\
f_{n}^{\alpha}\left(x_{\alpha}\right) \neq a_{\beta} \text { for any } n \in \mathbb{N} \text { and } \beta \leq \alpha .
\end{aligned}
$$

Set $A:=\left\{a_{\alpha}: \alpha<\kappa\right\}$. Then $A$ is not meager and for any sequence $\left(f_{n}: n \in \mathbb{N}\right)$ of continuous one-to-one functions from $\left(\{0,1\}^{\mathbb{N}}, \tau_{p}\right)$ into $[0,1],\left(f_{n}^{-1}(A): n \in \mathbb{N}\right)$ does not form a cover of $\{0,1\}^{\mathbb{N}}$. So, if we take $\mathcal{A}$ to be the $\sigma$-ideal generated by the inverse images, $f^{-1}(A)$, as $f$ runs over all the continuous one-to-one functions from $\{0,1\}^{\mathbb{N}}$ into $[0,1]$, then $\mathcal{A}$ will be a strongly topologically stable proper $\sigma$-ideal on $\left(\{0,1\}^{\mathbb{N}}, \tau_{p}\right)$ such that $C \cap A \in \mathcal{A}_{C}$ for every set $C \subseteq[0,1]$ that is homeomorphic to $\left(\{0,1\}^{\mathbb{N}}, \tau_{p}\right)$.

Corollary 2. There exists a Banach space $(X,\|\cdot\|)$ such that $\left(X^{*}\right.$, weak $\left.{ }^{*}\right)$ is weakly Stegall but $(X,\|\cdot\|)$ is not weak Asplund. In particular, $(X,\|\cdot\|)$ is a Gâteaux differentiability space that is not weak Asplund.

Proof. Let $A$ be the set constructed in Lemma 3 and let $\mathcal{A}$ be the corresponding $\sigma$-ideal on $\left(\{0,1\}^{\mathbb{N}}, \tau_{p}\right)$. Then $A$ satisfies the hypotheses of Theorem 4 with respect to $\mathcal{A}$. Hence $\left(B V_{A}[0,1], \tau_{p}\right)$ is nearly Stegall with respect to $\mathcal{A}$. Therefore, by Proposition $1,\left(C\left(K_{A}\right)^{*}\right.$, weak $\left.{ }^{*}\right)$ is nearly Stegall with respect to $\mathcal{A}$ and so weakly Stegall. On the other hand, if $\left(C\left(K_{A}\right),\|\cdot\|_{\infty}\right)$ is weak Asplund, then by [1, every closed subset of $K_{A}$ contains a dense completely metrizable subspace. However by Proposition 5 in [5] this implies $A$ is meager (in fact perfectly meager); which it is not. Therefore, $\left(C\left(K_{A}\right),\|\cdot\|_{\infty}\right)$ is not weak Asplund.

Remark. We note here that the statement of Theorem 4 can be recast in terms of the following definition. If $\mathcal{A}$ is a proper $\sigma$-ideal of subsets on $\{0,1\}^{\mathbb{N}}$ and $N$ is a subset of a complete metric space $M$, then we say that $N$ is $\mathcal{A}$-negligible if $\gamma^{-1}(N) \in \mathcal{A}$ for each $\gamma$ belonging to a residual subset $R_{N}$ of $C\left(\{0,1\}^{\mathbb{N}}, M\right)$; the continuous functions from $\{0,1\}^{\mathbb{N}}$ into $M$ equipped with the topology of uniform convergence. [Note: the residual set $R_{N}$ will in general depend upon the set $N$.]

Theorem 4 can then be rephrased as: "Let $\mathcal{A}$ be a proper $\sigma$-ideal on $\{0,1\}^{\mathbb{N}}$ and let $A$ be a dense subset of $(0,1)$ such that $\gamma^{-1}(A) \in \mathcal{A}$ for every homeomorphic embedding of $\left(\{0,1\}^{\mathbb{N}}, \tau_{p}\right)$ into $[0,1]$. Then for every complete metric space $M$ without isolated points and minimal usco $\varphi: M \rightarrow 2^{B V_{A}[0,1]}, \varphi$ is single-valued except on an $\mathcal{A}$-negligible subset of $M$. In particular, $\left(B V_{A}[0,1], \tau_{A}\right)$ is weakly Stegall”.

Let us also note that a subset $N$ of a complete metric space $M$ that has the Baire property is $\mathcal{A}$-negligible if and only if it is of the first category. Hence the interesting $\mathcal{A}$-negligible sets are necessarily among those sets that are not very topologically respectable. 


\section{ACKNOWLEDGEMENT}

The authors would like to thank both David Fremlin and David Preiss for (independently) bringing Lemma 3 to their attention. The authors would also like to thank Kenneth Kunen for some preliminary discussions concerning set theory.

\section{NOTE ADDED IN PROOF}

Since this manuscript was first submitted in 2002, a simplified proof of Corollary 2 has appeared in 8 .

\section{REFERENCES}

1. M. M. Čoban and P. S. Kenderov, Generic Gâteaux differentiability of convex functionals in $C(T)$ and the topological properties of $T$, Proceedings of 15th Spring Conference of the Union of Bulgarian Mathematicians, Sljanĉev Brjag, (1986), 141-149. MR0872913 (88d:46037)

2. M. J. Fabian, Gâteaux differentiability of convex functions and topology: Weak Asplund spaces, Canadian Mathematical Society Series of Monographs and Advanced Texts. WileyInterscience, New York, 1997. MR 1461271 (98h:46009)

3. J. R. Giles, Convex Analysis with Application in Differentiation of Convex Functions, Research Notes in Mathematics, 58 Pitman, Melbourne, 1982. MR0650456 (83g:46001)

4. O. F. K. Kalenda, Weak Stegall spaces, unpublished manuscript, Spring 1997 (3 pages).

5. O. F. K. Kalenda, Stegall compact spaces which are not fragmentable, Topology Appl. 96 (1999), 121-132. MR.1702306 (2000i:54027)

6. P. S. Kenderov, W. B. Moors and S. Sciffer, A weak Asplund space whose dual is not weak* fragmentable, Proc. Amer. Math. Soc. 129 (2001), 3741-3747. MR1860511(2002h:54014)

7. D. G. Larman and R. R. Phelps, Gâteaux differentiability of convex functions on Banach spaces, J. London Math. Soc. 20 (1979), 115-127. MR0545208 (80m:46017)

8. W. B. Moors, Some more recent results concerning weak Asplund spaces, Abstr. Appl. Anal. 2005 (2005), 307-318. MR2197122

9. W. B. Moors and S. Somasundaram, Some recent results concerning weak Asplund spaces, Acta Univ. Carolin. Math. Phys. 43 (2002), 67-86. MR1979559(2004e:46027)

10. R. R. Phelps, Convex functions, monotone operators and differentiability, Lecture notes in Mathematics, Springer-Verlag, Berlin, 1993. MR1238715 (94f:46055)

11. C. Stegall, A class of topological spaces and differentiability, Vorlesungen aus dem Fachbereich Mathematik der Universität Essen 10 (1983), 63-77. MR0730947 (85j:46026)

12. C. Stegall, The topology of certain spaces of measures, Topology Appl. 41 (1991), 73-112. MR 1129700 (93d:46067)

Department of Mathematics, The University of Auckland, Private Bag 92019, AuckLAND, NeW ZEALAND

E-mail address: moors@math.auckland.ac.nz

Department of Mathematics, The University of Waikato, Private Bag 3105, Hamilton 2001, New ZeALAND

E-mail address: ss15@math.waikato.ac.nz 